\title{
Oily Wastewater Treatment Using Membranes Modified by Plasma
}

\author{
Dryahlov Vladislav Olegovich ${ }^{1, *}$, Shaihiev Ildar Gilmanovich ${ }^{1}$, Bogdan Stoyanov Bonev ${ }^{2}$, Abdullin Ildar \\ Shaukatovich ${ }^{1}$, Valentin Andreev Nenov ${ }^{2}$
}

${ }^{1}$ The Kazan national Research Technological University,Russian Federation, Kazan, Karl Marx street 68.

${ }^{2}$ Bulgaria, Burgas, boulevard Professor Yakimov 1.

*Corresponding Author: vladisloved@mail.ru

Copyright (C) 2013 Horizon Research Publishing All rights reserved.

\begin{abstract}
The influence of parameters of low-temperature high-frequency plasma of low pressure on the efficiency of emulsions division in the sort of "oil-in water" was investigated using polyacrylonitrile membranes with pores about $50 \mathrm{kDa}$. It was indicated, that plasma treatment of membranes in an atmosphere of argon with nitrogen promotes the increase in efficiency and selectivity of division more than twofold. The investigations of surface characteristics of initial and plasma-treated membranes were carried out by the instrumental controls of analysis. It was indicated, that plasma treatment promotes structure changes of membrane surface, without changing the internal structure of membrane polymer.
\end{abstract}

Keywords Division, Membranes, Plasma, Modification

Oil-In Water Emulsion

\section{Introduction}

Lubricating fluids take the special place among a great number of process liquids used at power engineering, mechanic engineering and metallurgic engineering enterprises. It is aggregative and kinetic stable emulsions that are type of "oil-in water", having used in the process of metal working for the purpose of temperature reduction and increase of machining tools wear resistance. In the operational maintenance, lubricant mediums are polluted by different impurities, are subjected to bio-affect, and loose the technological potential. This leads up to necessity of frequent change of lubricating fluids by fresh doses and discharge liquids are fed to purification plants. Owing to complexity of multicomponent composition, stability to influence of microorganisms and the necessity of emulsion stable structure disruption, traditional methods of clarification, filtering or biological treatment can't be enough effective.
There is a great number of recovery processes, recuperation and regeneration of lubricating fluids, but the most popular method is using membrane technologies [1-3]. This fact is related to the next advantages of the last: the division of liquid mixtures proceeds without phase conversions that supposes low energy demands; the process proceeds at temperatures approximate to environmental temperature, that is very important for mixture division, which components can be decomposed and wrecked or polymerized at higher temperatures, reside in traditional technical methods; turbidness and colour of water are decreased essentially; required space for facilities is less of the installations squire of mechanic, chemical and biological treatments; facilities are simple in the construction and reliable in operations.

The low capacity and necessity of preliminaries, connected to disposal of dispersion systems with the size more than $20 \mathrm{mkm}$ and of neutralization of aggressive mediums should be attributed to demerits of membrane processes.

Due to above-mentioned disadvantages, it is to be noted that researches in the modification sphere of membranes surface texture are carried out very active for the last time in order to increase productivity and efficiency of division. The special success was achieved in the modification by chemical reagents and high frequency capacitive plasma (HFC) [4-6].

Plasma represents the mixture of neutral atoms and molecules, excited electrons and ions. As far as majority of polymers are dielectrics and the base for membranes preparation, high frequency field can pass through them, self-maintained discharge sets in pores and treatment of both the surface and the internal structure of membrane occurs. The following processes make a main contribution to modification of polymers: kinetic impact of low energy ions of plasma $(\mathrm{E}=10-100 \mathrm{eV})$; ion recombination on the surface of capillary porous bodies; minor thermal influence (from 25 till $60{ }^{\circ} \mathrm{C}$ ). Previous investigations proved that it is possible to change mechanical and technological membrane characteristics, which includes hydrophilicity and hydrophobicity by varying data of plasma treatment [7]. 


\section{Methodology}

In view of the foregoing, researches of water and oil emulsion divisions were conducted by means of initial and plasma treated membranes with the pore size of $50 \mathrm{kDa}$, which were made from polyacrylonitrile (PAN). The treatment was done by low temperature HFC plasma of low pressure in the atmosphere of argon and nitrogen (hydrophilous regime) and propane and butane (hydrophobous regime) in the ratio 70:30 in accordance with the following conditions: current intensity on anode is $\left(\mathrm{I}_{\mathrm{a}}\right)-$ $0.5 \mathrm{~A}$, mixed gas rate $(\mathrm{G})$ is $-0.04 \mathrm{~g} / \mathrm{sec}$, pressure $(\mathrm{P})$ is 26.6 $\mathrm{Pa}$. In the course of membranes treatment by plasma flow, plasma action time $(\tau)$ differed $1.5,4$ and $7 \mathrm{~min}$ and voltage on plasmatron anode $\left(\mathrm{U}_{\mathrm{a}}\right)$ varied from 1.5 till $7.5 \mathrm{kV}$.

Qualitative and quantitative composition of emulsion is the following: industrial oil «I-20A» is - $20 \%$, surface-active substance (SAS) «Kosintanol 242» is - $2 \%$, distilled water is $-78 \%$.

The research of emulsion division was done in the laboratory facility using the «Cross-flow» process in applying ambient pressure $202.65 \mathrm{~Pa}$ and temperature $20{ }^{\circ} \mathrm{C}$. Productivity and efficiency factors were considered as the main parameters of membrane systems operation. The first index is the ratio of quantity of passed through membrane stream of shared medium to the time product and the filtration area, which is $1.73 \cdot 10-3 \mathrm{~m}^{2}$ in this case. The efficiency factor was estimated according to index change of chemical oxygen demand (COD) and oil content in the treated wastewater, measured by autotitrator «T70» and concentratometer $\langle\mathrm{KN}-2 »$ accordingly.

\section{Results and Discussions}

Productivity figures are presented at the figure 1 in the form of diagrams. The analysis of the graphic chart introduced in the figure 1 , shows that productivity increases twice in the result of membranes treatment by plasma in the nitrogen and argon medium.
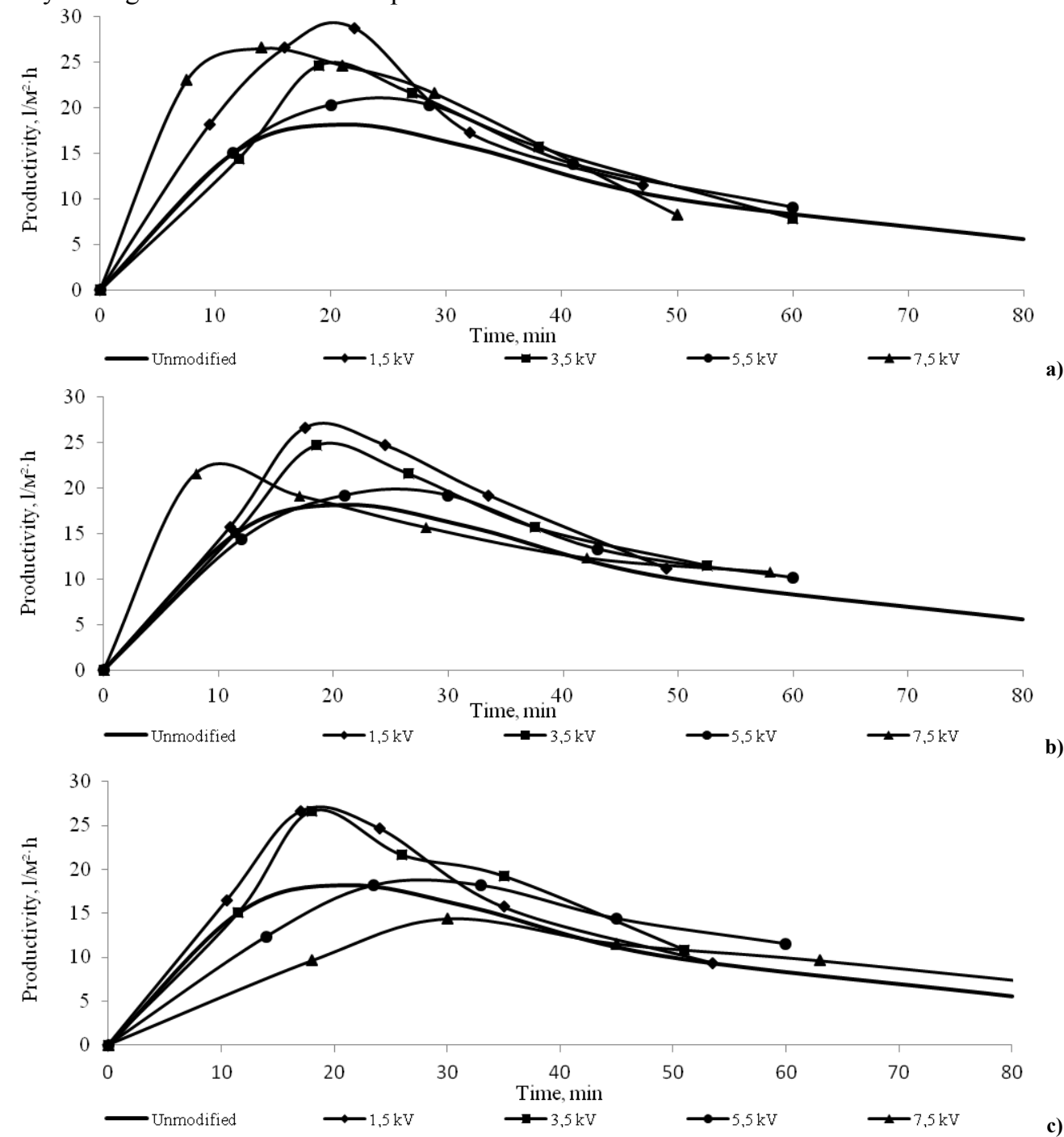

b)

Figure 1. Characteristic curve of membrane productivity in the dependence of anode voltage value and plasma treatment time: a) 1.5 min; b) 4 min; c) 7 min. 
COD indexes and oil content in the treated wastewater, obtained in the process of emulsions division, are presented in the table 1 and table 2 accordingly.

Table 1. COD filtrates indexes, obtained in the process of emulsions division using initial and plasma-treated membranes.

\begin{tabular}{|c|c|c|c|}
\hline \multirow{2}{*}{$\begin{array}{c}\text { Anode voltage of } \\
\text { plasmatron }\left(\mathrm{U}_{\mathrm{a}}\right)\end{array}$} & \multicolumn{3}{|c|}{ Treatment time } \\
\cline { 2 - 4 } $\mathrm{kV}$ & $1.5 \mathrm{~min}$ & $4 \mathrm{~min}$ & $7 \mathrm{~min}$ \\
\cline { 2 - 4 } & \multicolumn{2}{|c|}{ COD filtrates indexes, $\mathrm{mg} \mathrm{O}_{2} / 1$} \\
\hline 1.5 & 5810 & 9360 & 12930 \\
\hline 3.5 & 5700 & 2700 & 9240 \\
\hline 5,5 & 11240 & 8900 & 10810 \\
\hline 7.5 & 5760 & 5520 & 4720 \\
\hline Initial membrane & & 12100 \\
\hline
\end{tabular}

Table 2. Oil content in the treated wastewater, obtained in the process of emulsions division using initial and plasma-treated membranes.

\begin{tabular}{|c|c|c|c|}
\hline \multirow{2}{*}{$\begin{array}{c}\text { Anode voltage of } \\
\text { plasmatron }\left(U_{\mathrm{a}}\right), \\
\mathrm{kV}\end{array}$} & \multicolumn{3}{|c|}{ Treatment time } \\
\cline { 2 - 4 } & $1.5 \mathrm{~min}$ & $4 \mathrm{~min}$ & $7 \mathrm{~min}$ \\
\cline { 2 - 4 } & Oil content in the treated wastewater, $\mathrm{mg} / \mathrm{l}$ \\
\hline 1.5 & 8803 & 13370 & 21550 \\
\hline 3.5 & 8380 & 4426 & 14660 \\
\hline 5,5 & 17560 & 12350 & 17160 \\
\hline 7.5 & 8860 & 8120 & 6849 \\
\hline Initial membrane & \multicolumn{3}{|c|}{18330} \\
\hline
\end{tabular}

Filtrate and concentrate are formed in the result of division of initial test emulsion, whose index COD and oil content was equaled $147900 \mathrm{mg} \mathrm{O} / \mathrm{l}$ and $224000 \mathrm{mg} / \mathrm{l}$ accordingly by means of initial unmodified PAN membrane. Indexes COD and oil content in the treated wastewater and concentrate were equaled 12100 and $267100 \mathrm{mg} \mathrm{O}_{2} / 1,18330$ and $404700 \mathrm{mg} / \mathrm{l}$ accordingly. Hence, general effectiveness of separating process is as follows $91.8 \%$.

The resulting concentrate had a moisture content of less than $30 \%$. Therefore, it can be utilized to provide secondary combustion heat [7].

The data shown in the table 1 confirm that effectiveness of the division process of water - in - oil medium improved due to membrane treatment by plasma in argon and nitrogen medium. This is confirmed by index declination of COD filtrates in 2-3 times, which were obtained with the use of plasma modified filter-elements in comparison with initial ones. However, definite characteristic curves of the observed index from plasma treatment parameters were not revealed. The lower range value $\mathrm{COD}=2700 \mathrm{mg} \mathrm{O} / 1$ was obtained by using membrane, treated in the plasma stream during 4 minutes with anode supply voltage in $3.5 \mathrm{kV}$. As a result of before-mentioned, the total effectiveness equaled $98.2 \%$.

As a result, it was shown, that PAN membranes treatment HFC of low temperature plasma of low pressure in the argon and nitrogen medium leads to productivity and effectiveness increase of breaking of water-in oil emulsion. Carried out experiments approved, that division of test emulsion doesn't happen in the result of membranes treatment in the propane-butane medium in this connection, given filter-elements were not used in researches.

The data presented in Table 2 confirm the findings of the analysis data of Table 1 .

Investigations of surface and structural characteristics of initial and plasma-treated membranes in the medium of argon and nitrogen, propane and butane in the ratio $70: 30$ for $\mathrm{Ua}=5.5 \mathrm{kV}$ and $\tau=4 \min (\mathrm{Ia}=0.5 \mathrm{~A}, \mathrm{G}=0.04 \mathrm{~g} / \mathrm{sec}, \mathrm{P}=$ $26.6 \mathrm{~Pa}$ ) were carried out by methods of electron microscopy, measurement of interfacial angle watering and $\mathrm{X}$-ray crystal analysis (XCA) with the aim of explanation of the above mentioned circumstances.

Surface images and histograms of initial and plasma-treated membranes, obtained with the aid of scanning probe microscope «MultiMode $V »$ were presented in the figure 2.
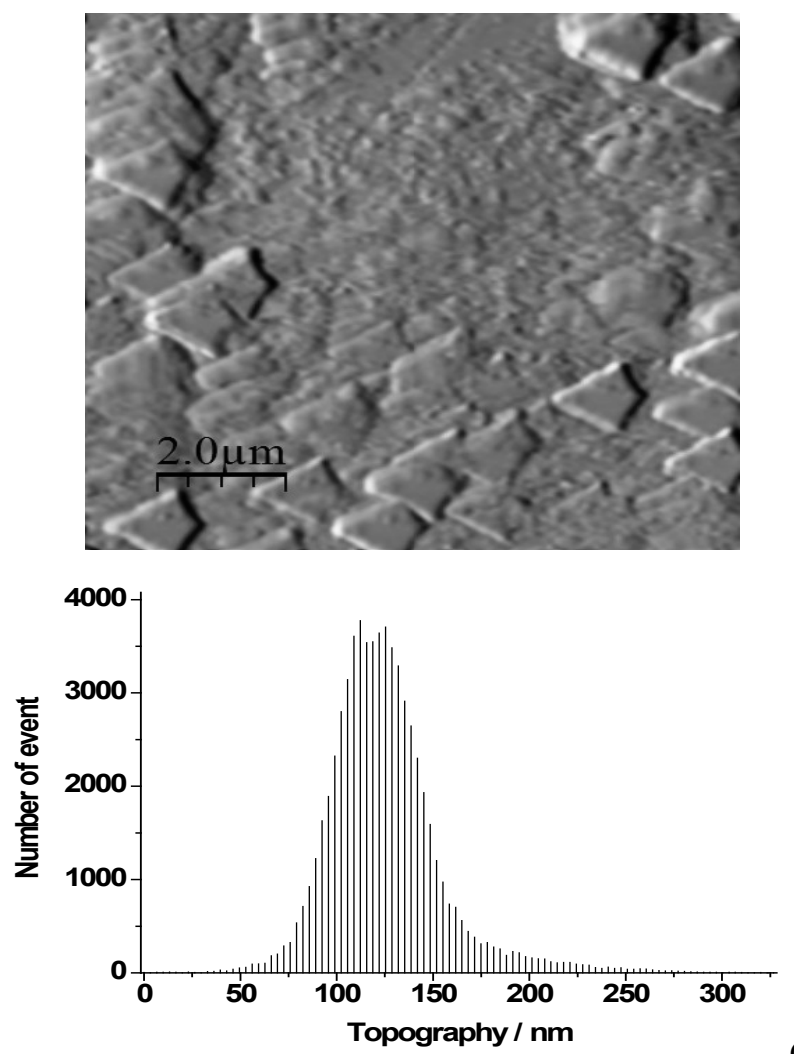

(a)

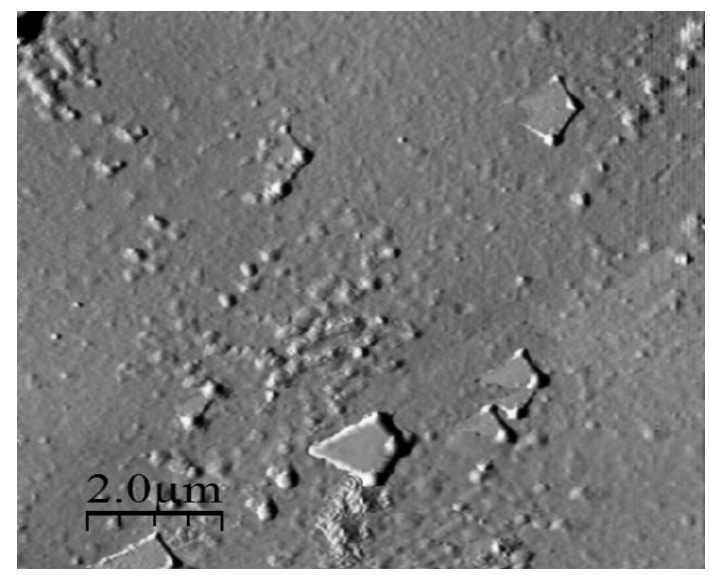




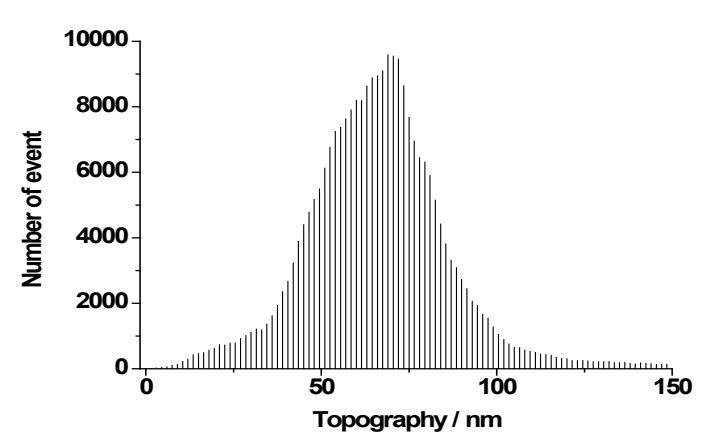

(b)
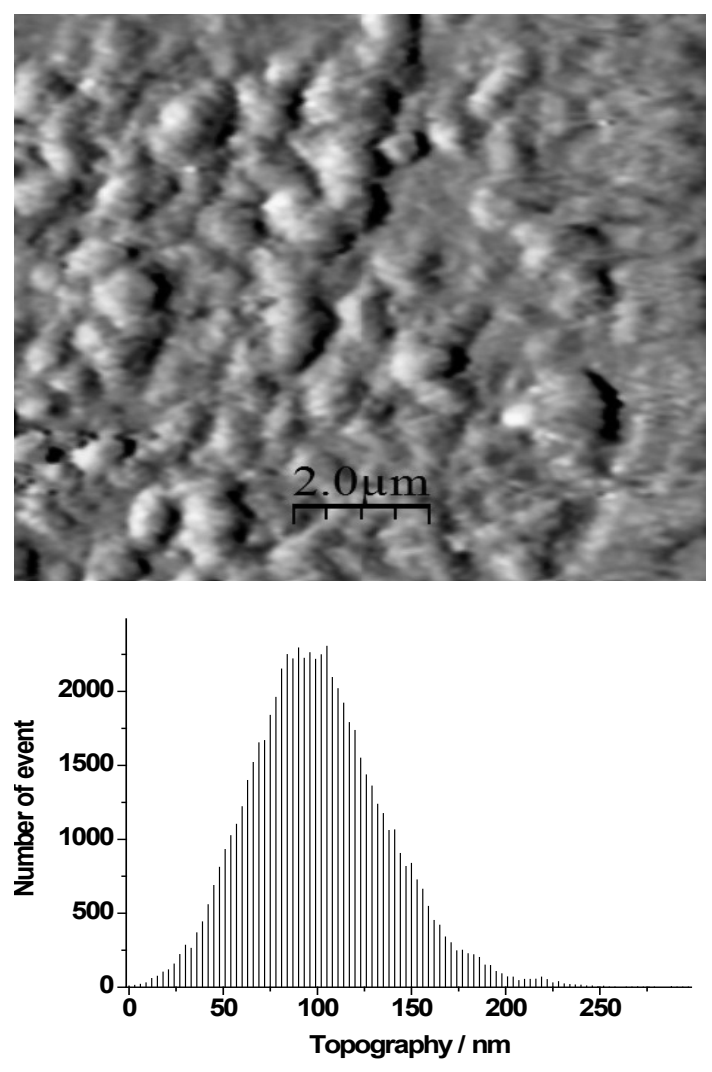

(c)

Figure 2. Images of surfaces and histograms of PAN membranes: a) unmodified; b) processed in argon and nitrogen environment; c) processed in propane and butane environment.

The existence of surface changes of plasma-modified membranes (fig. 2b and 2c) in comparison with the initial ones (fig. 2a) was determined in the result of imaging analysis, presented in the figure 2 . This circumstance is the result of different physico-chemical processes in the course of plasma treatment, such as the process of etching, oxidation and oxidation etching, disrupture and crossing, bond opening with forming polar groups when dealing with gas phase of plasma and et.cent. It is impossible to devide these processes by consecutive stages. The process of plasma-chemical modification is multi-channel and as a rule, mentioned above its directions coexist with the single result at the same time. This result is the change of the structure and surface characteristics of polymer material.
Presented histograms in the fig. 2 are the graphic chart of pimples allocation on the membrane surface, where the pimples height in $\mathrm{nm}$ is situated across and the ratio with given lapse rate is vertically. Degradation of pimples height on the surface of PAN microelements takes place in the result of HFC plasma influence. Maximum dimension of pimples on the surface of unmodified membrane totaled 120-125 nm, just as maximum dimension of pimples on the membranes surface of processed in argone and nitrogen, propane and butane medium totalled 65-70 and 105-110 nm properly.

Hence, the variance of surface characteristics of plasma processed membranes owing to above discribed physical and chemical reactions behavior was shown and proved quantatively.

The more important on the practical level result of low temperature plasma influence on polymer materials is changing their adhesive properties. Under the action of plasma, the polymer surface can become as more hydrophilous as more hydrophobous. [8].

In confirmation of before mentioned, images of a distilled water drop on the surface of initial and plasma processed membranes and as well as values of limiting wetting angle are presented in the figure 3 .

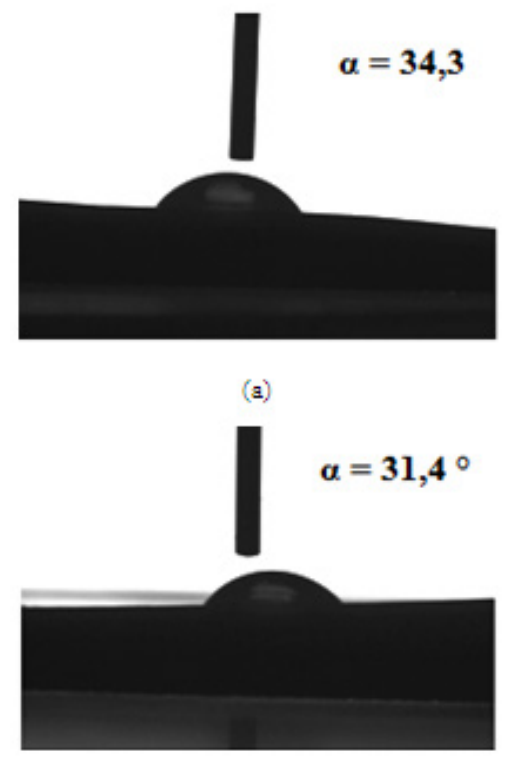

(b)

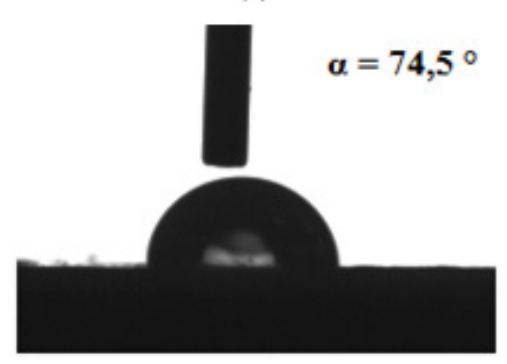

(c)

Figure 3. Images of limiting wetting angle of membrane surface by a distilled water drop: a) unmodified; b) processed in argon and nitrogen medium; c) processed in propane and butane medium. 
In the result of plasma treatment of initial PAN membrane, the value of limiting wetting angle by the a distilled water drop is $\alpha=34.3^{\circ}$ (fig. 3a). Degradation of considerable parameter takes place in argon and nitrogen medium till $\alpha=$ $31.4{ }^{\circ}$. Evidently, that the surface becomes more hydrophilous (fig. 3b), the membrane surface obtains more hydrophobous character in the case of propane and butane influence, limiting wetting angle increases till $\alpha=74.5^{\circ}$ (fig. $3 \mathrm{c})$.

In such a way, more hydrophilous membrane shows more selectivity with regards to water. As a result, productivity increases and the index of COD filtrates, obtained when using filter-elements, treated by plasma in the atmosphere of argon and nitrogen decreases. Repulsion of water molecules by the hydrophobous surface happens when using a hydrophobous membrane. As a result, the division of waterin - oil emulsion doesn't take place.

The dynamic of water-wetting by the distilled water drop of the surface of initial and plasma treated membranes is presented in the figure 4 .

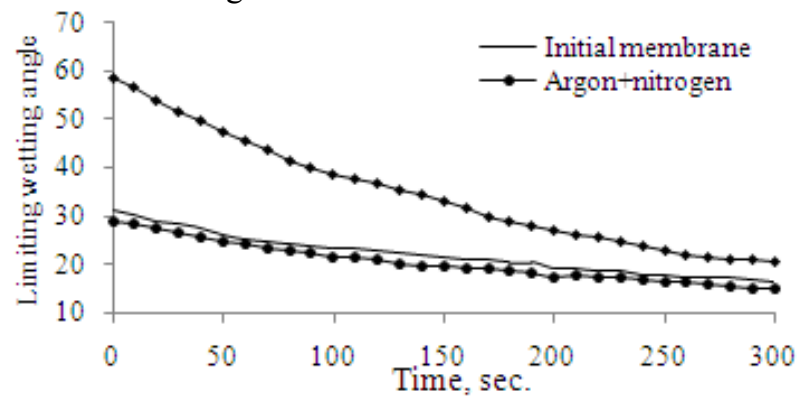

Figure 4. The dynamic of water-wetting by the distilled water drop of membranes surface

The limiting wetting angle decreases eventually as is shown by data in the figure 4

It is known about interdependency of water-wetting parameters and degrees of polymers crystallinity from literary sources [8]. Diffractograms of initial and plasma treated membranes with adequate data of crystallinity degree $(\mathrm{Y})$ are presented in the figure 5.

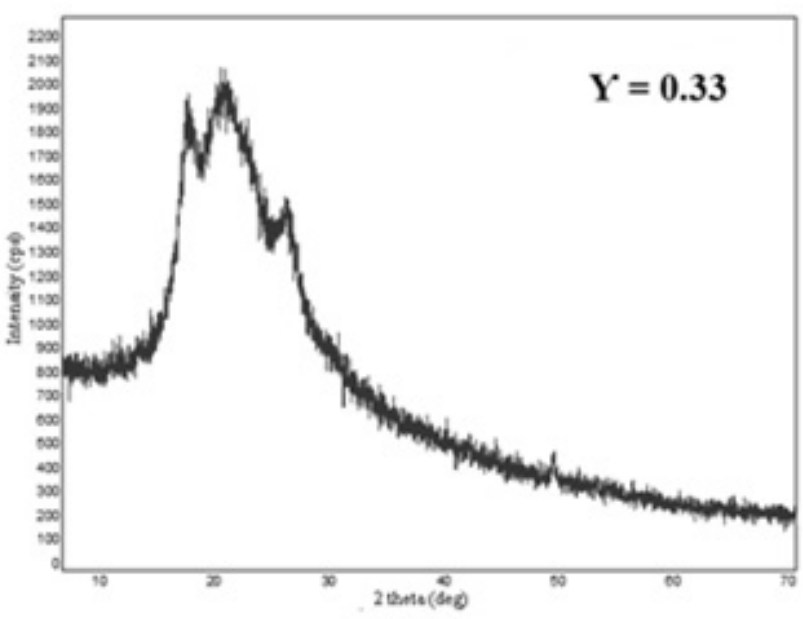

(a)

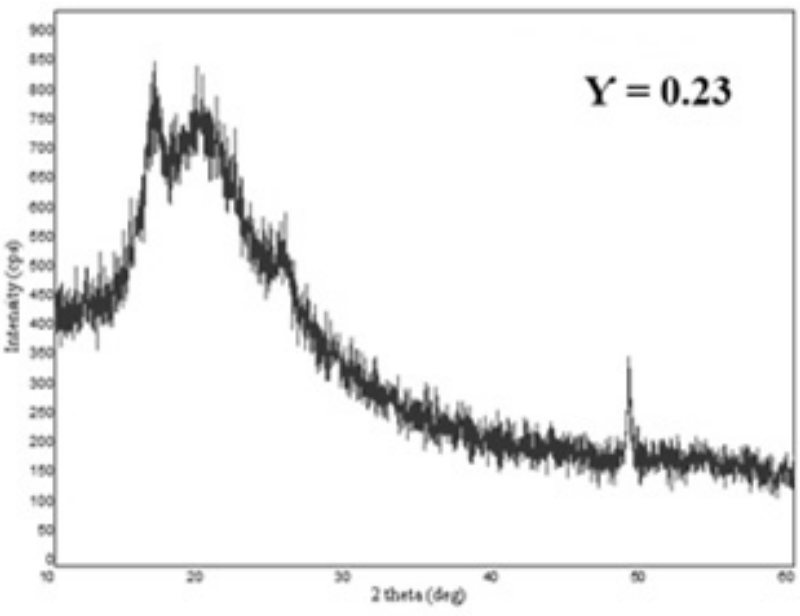

(b)

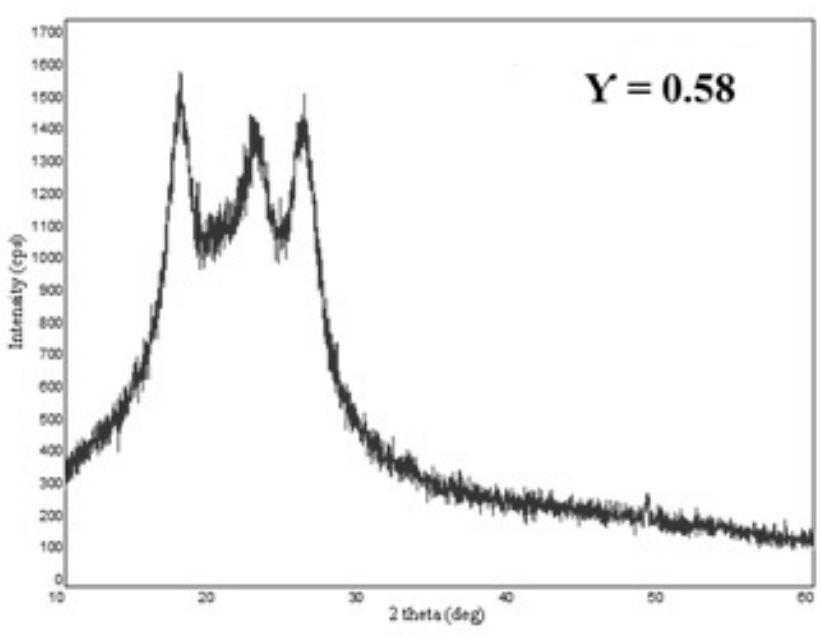

(c)

Figure 5. Difractograms of PAN membranes: a) unmodified; b) treated in argon and nitrogen medium; c) treated in propane and butane medium.

In the result of data analysis, analogous to previous research dependencies were determined and presented in the figure 5: decrease of crystallinity degree takes place in the result of PAN membranes treatment by plasma in hydrophilous regime similar to values of the limiting wetting angle. And crystallinity degree increases in the case of treatment in the hydrophobous regime. This fact lets confirm some correlation degree between water-wetting parameters and crystallinity degree that gives the opportunity for further purposeful modification of PAN membranes in order to achieve required characteristics.

It is important to note, that plasma treatment changes only the surface structure of PAN membranes. It is documented by the above mentioned data and it doesn't influence on the polymer structure, which was the base for making filter element. Hence, for example, IR-spectrum of the initial membrane is absolutely identical to the same spectrum of plasma treated membrane. 


\section{Conclusion}

Therefore, the presence of surface deformations, changes of adhesion properties and crystallinity degree of modified membranes, affecting the productivity and selectivity of PAN membranes in the process of emulsion division of water-in-oil type and depending on plasma treatment modes were determined by the results of instrumental methods.

Modification practicability of PAN membranes by low temperature high frequency capacitance plasma of low pressure was shown by the experimental data with the aim to increase efficiency of water-in-oil emulsion division, which is the main prototype of lubricating fluids and is widely used in industry.

\section{REFERENCES}

[1] N. Drouiche, M. Naceur, H. Boutoumi, N. Aitmessaoudene, R. Henniche, T. Ouslimane. Assessment of the recovery of photovoltaic cells cutting fluid by chemical pretreatment and ultrafiltration, Desalination and Water Treatment, Vol.51, No.5, 713-716, 2013.

[2] J. Wu, W. Jing, W. Xing, N. Xu. Preparation of w/o emulsions by membrane emulsification with a mullite ceramic membrane, Desalination and Water Treatment, Vol.21, No.2, 381-386, 2006.

[3] W. Chen, J. Peng, Y. Su, L. Zheng, L. Wang, Z. Jiang. Separation of oil/water emulsion using pluronic f127 modified polyethersulfone ultrafiltration membranes, Separation and purification technology, No.66, 591-597, 2009.

[4] E. Finot, S. Roualdes, M. Kirchner, V. Rouessac, R. Berjoan, J. Durand, J. Goudonnet, L. Cot. Surface investigation of plasma hmdso membranes post-treated by cf4/ar plasma, Applied surface science, Vol.15, No187, 326-338, 2002.

[5] P. Wanichapichart, R. Sungkum, W. Taweepreda, M. Nisoa. Characteristics of chitosan membranes modified by argon plasmas, Surface and coatings technology, Vol.203, 2531-2535, 2009.

[6] K. Kull, M. Steen, E. Fisher. Surface modification with nitrogen-containing plasmas to produce hydrophilic, low-fouling membranes, Journal of membrane science, Vol.246, 203-215, 2005.

[7] V. Dryahlov, I. Shayhiev, I. Abdullin, R. Ibragimov, R. Batyrshin. Research of dividing of water-oil emulsions with the help of membranes modified by plasma, Vestnik KTU, No.11, 43-48, 2011.

[8] G. Slonimsky. Modern Physical Methods of Research of Polymer, Chemistry Publication, Moscow, 1982. 